\title{
RECONSTRUCTION OF POSTERIOR MEATAL AND/OR LATERAL ATTIC WALLS IN CHOLESTEATOMA SURGERY
}

\author{
Mohammed Elsayed Elmaghawry, Mohammed Kamal Mobasher, Magdy Mohammed Abd-Elfattah, Adly \\ Ahmed Tantawy,Atef Hamed Ali \& Ahmed Mohammed Anany \\ Department of Otorhinolaryngology, Zagazig University Hospitals,Zagazig,Egypt
}

\begin{abstract}
Purpose: To evaluate reconstruction of posterior meatal and/or lateral attic walls in cholesteatoma surgery using the autologous bony posterior canal wall and bone pâté during the first stage operation.

Patients and methods: Twenty ears of twenty patients with chronic suppurative otitis media with cholesteatoma. Cases with extensive destruction of posterior canal wall and mastoid cortex, low tegmen, significant anterior or lateral sigmoid sinus, only functioning ear, labyrinthine fistula and previous canal wall down mastoidectomy were excluded. The surgical technique includes cortical mastoidectomy and bone pâté collection,temporary removal of the bony posterosuperior meatal wall(PMW) by cutting the entire PMW as one piece with a microsagittal saw to offer optimal exposure of tympanic cavity,attic and retrotympanum for complete eradication of cholesteatoma and then repositioning of this wall in its anatomical site supported with bone pâte in attic and mastoid.The condition of reconstructed PMW and the status of middle ear were evaluated by computed tomography scan, otoendoscopic examination and staged second-look surgery.

Results:Cholesteatoma in the mastoid, antrum, attic and retrotympanum could be removed with safety in all cases; no serous intraoperative complications occurred, intraoperative difficulties included: damage of posterosuperior canal wall during saw cutting in one case $(5 \%)$ and mild dural injury during superior cutting in one case(5\%), through follow-up , the new reconstructed canal appeared to be of near normal size, shape and contour, and no dislocation or necrosis of the reconstructed posterior canal wall was noted. The postoperative complications included: wound infection in one case $(5 \%)$, tinnitus in two cases $(10 \%)$ and recurrent cholestearoma in one case $(5 \%)$.

Conclusion: Our surgical technique which includes reconstruction of posterior meatal and/or lateral attic walls in cholesteatoma surgery using the autologous bony posterosuperior meatal wall after its temporary removal provides optimal surgical exposure during the procedure, restores near normal anatomy of the external auditory canal, has low recurrence rate $(5 \%)$ and avoids the troubles of open mastoid cavity, making this surgical procedure an attractive alternative to the standard CWU and CWD procedures.

Keywords:Cholesteatoma; Canal wall down mastoidectomy; Canal wall up mastoidectomy; Posterior canal wall reconstruction; Open cavity.
\end{abstract}

\section{INTRODUCTION}

Cholesteatoma is a surgical disease for which the primary universally goal is total eradication of cholesteatoma to obtain a safe and dry ear, the second objective is restoration or maintaining the functional capacity of the ear, the hearing. The Third objective is to maintain a normal anatomic appearance of the ear if possible (1).Surgical management of cholesteatoma remains a controversial issue. The canal wall down (CWD) mastoidectomy represents the classic procedure For the removal of cholesteatoma from the epi-and retrotympanal spaces. With this open technique, the incidence of residual and recurrent cholesteatoma can be effectively prevented due to excellent exposure of all middle ear spaces (2). The disadvantages of the open technique include; the problems related to the wide mastoid cavity that needs regular lifelong otologic care for the debris, propensity toward caloric stimulation by cold water or air and difficulties in the placement of hearing- aid devices when needed(3). To avoid these problems,there has been a trend to maintain the normal anatomy of the ear canal by canal wall up (CWU) techniques. Unfortunately CWU techniques have resulted in a high rate of residual cholesteatoma up to $60-70 \%$ because the surgical exposure and access to all tympanic cavity and retrotympanic spaces is greatly limited and impeded(4). The advantages of both techniques (CWD \& CWU) - optimal exposure of all middle ear spaces and preservation of the normal anatomy- are offered by procedures that involve the temporary removal and reinsertion(5) or reconstruction of the posterior canal wall(6). The purpose of this study was to evaluate the out-come of reconstruction of posterior meatal and / or lateral attic walls in cholesteatoma surgery by using the autologous bony posterosuperior meatal wall and bone pâté.

\section{PATIENTS AND METHODS}

This study was carried out on 20 ears of 20 patients in Department of Otorhinolaryngology, Head and Neck Surgery, Zagazig University Hospitals from September 2011 through March 2014. The patients consisted of 14 females and 6 males, their ages ranged from 14 to 52 years. All patients suffered from chronic otitis media with cholesteatoma. The cases with extensive destruction of posterior canal wall and mastoid cortex, low tegmen, significant anterior or lateral sigmoid sinus, only functioning ear, previous 
canal wall down procedure and labyrinthine fistula were excluded.

\section{Exclusion criteria of the patients :}

1- Extensive destruction of posterior canal wall and mastoid cortex.

2- Low tegmen.

3- Significant anterior or lateral sigmoid sinus.

4- Only functioning ear.

5- Previous canal wall down mastoidectomy.

6- Labyrinthine fistula.

The presenting complaints were persistent offensive ear discharge in all cases and associated variable hearing loss in 9 cases. Preoperative history taking, otomicroscopic and otoendoscopic ear examination and routine laboratory investigations were done in all cases. Preoperative audiological assessment (Pure Tone Audiogram) was done and all cases showed conductive hearing loss with air bone gaps (ABG) ranged from 10 to $45 \mathrm{dBHL}$. Pre operative high resolution computed tomography scanning

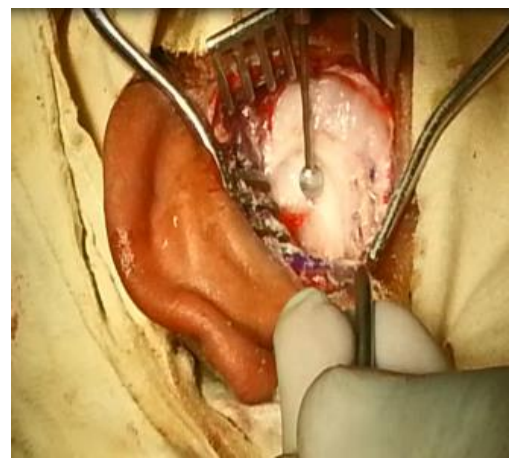

(A)

Figure (1):(A)Bone pâté collection from mastoid cortex, pâté after filtration.

Complete cortical mastoidectomy was preformed including total exenteration of the sinudural angle and mastoid tip air cells, and the posterosuperior bony canal wall was left relatively thick. The facial recess was opened and extended inferiorly to the level of the floor of the external auditory canal. The incus and malleus were removed and

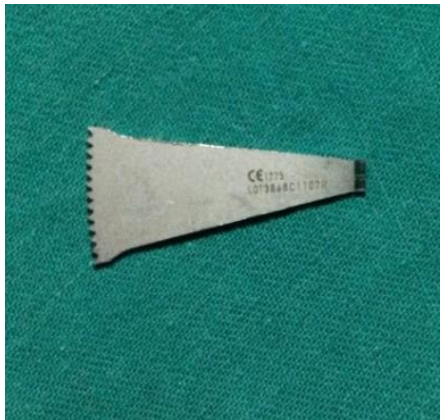

(A)

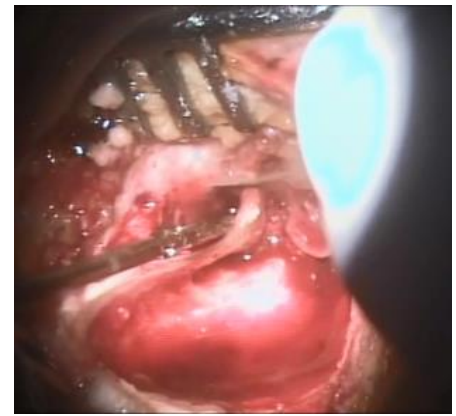

(B)
(HRCT scan) of the temporal bone was acquired in both axial and coronal planes in all cases.

Surgical Technique: All cases were operated under general anesthesia. Intraoperative parenteral antibiotic $\left(3^{\text {rd }}\right.$ generation cephalosporin) was given to all patients. The procedure was started with a traditional post auricular incision down to the level of the superficial layer of the deep temporalis fascia. An anteriorly based wide musculoperiosteal palva flap was created. The external canal skin was elevated off the posterior canal without making any incision in the skin. The annulus was elevated out of the annular sulcus. Care was taken to raise the canal wall skin anteriorly to protect it from the posterior bony canal wall cuts. Bone pâté collector was used to collect the bone pâté from the cortex of the mastoid and squamous temporal bone. Bone pâté collection stopped before exposure of the mastoid air cells. Saline solution was used to wash the bone pâté which was filtered through a piece of gauze and the bone pâté was set aside.

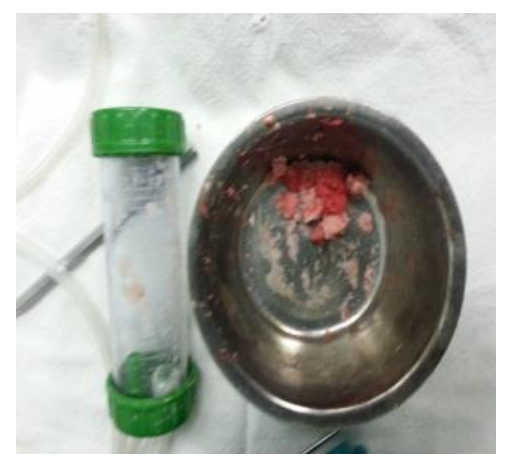

(B)

(B) Bone pâté collector and the collected bone

the chorda tympani nerve was scarified. The microsagittal saw was used to create superior and inferior cuts in the bony posterosuperior canal wall, the superior cut was parallel to the temporal bone and the inferior cut extended medially to the inferior facial recess.

Figure (2): Microsagittal saw (A), the saw used to create superior (B), and inferior(C) cuts in the posterosuperior canal wall. 
Then, the bony posterior canal wall segment was removed and placed aside for further reconstruction. The canal wall piece carefully examined to ensure all cholesteatoma and squamous epithelium had been removed.

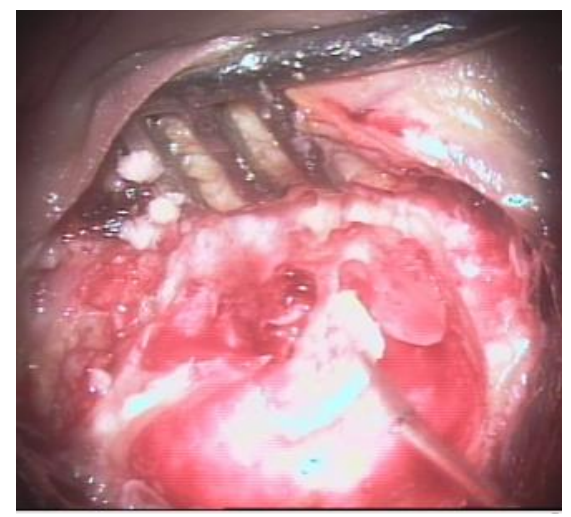

(A)

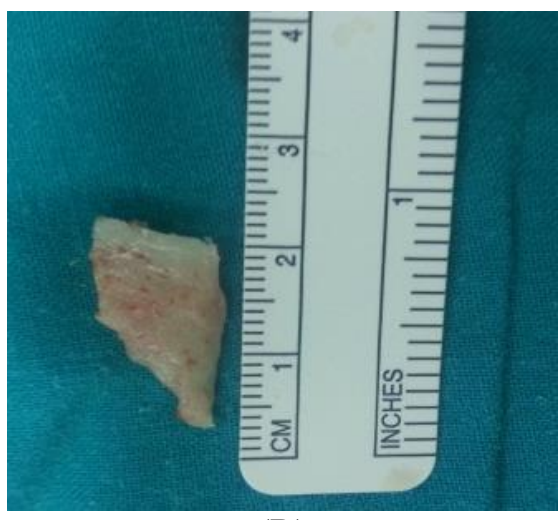

(B)

Figure (3): (A) Removal of the cutted posterior meatal wall, (B) Canal wall segment is placed aside for further reconstruction.

Cholesteatoma then completely removed from the tympanic cavity ,retrotympanum, mastoid and anterior attic. Generous temporalis fascia graft was harvested which must be large enough to extend up the posterior canal wall, over the canal wall cuts and used to reconstruct the tympanic membrane. The posterior canal wall segment was then replaced in its anatomical position and the bone pâté was carefully placed in the attic and mastoid to hold the canal wall segment in place and obliteration of mastoid and attic.

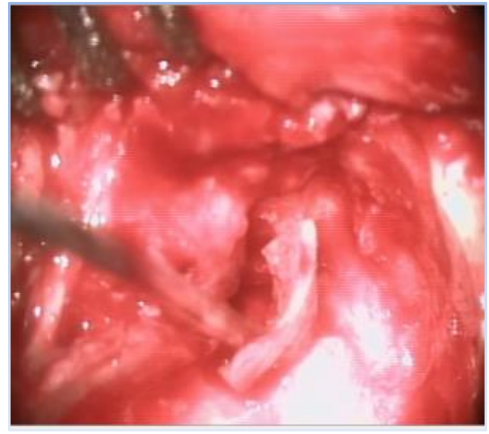

(A)

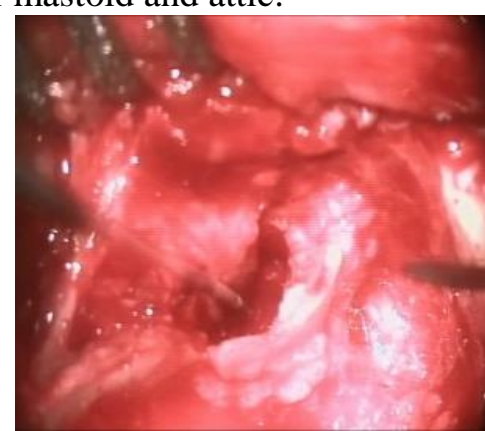

(B)

Figure (4): Repositioning of posterior meatal wall (A), and further support with bone pâté in attic and mastoid.

The temporalis fascia graft was used to reconstruct the tympanic membrane which extended up the reconstructed posterosuperior canal wall, over the canal wall cuts. The skin of the external auditory canal was repositioned on the temporalis fascia graft and the reconstructed posterosuperior canal wall. This was packed by several pieces of gel foam. The palva flap was closed with vicryl sutures. The post auricular incision was closed in two layers by interrupted sutures. The external auditory canal and meatus were packed with a gauze strip impregnated with antibiotic ointment. A standard mastoid dressing was applied. Reconstruction of the ossicular chain to restore the hearing mechanism was performed during the initial surgery in 6 cases, using partial ossicular replacement prosthesis (PORP) in 2 cases and cartilage graft over the stapes suprastructure in 4 cases.
Postoperative Care:All patients were discharged home in the operative day with oral antibiotic (ciprofloxacin in adults or amoxicillin clavulanate in patients under 18 years old) to complete 14 day course and oral analgesic. The mastoid dressing, canal pack and wound sutures were removed after one week postoperatively. Antibiotic ear drops were instilled until follow up after another 2 weeks (3 weeks postoperatively). The patients were instructed to keep the external auditory canal dry.

Patients follow -up: All patients were followed up once weekly in the first 2 months postoperatively, then once monthly. The duration of follow up ranged from 7 to 19 months. The follow up was done by history taking ,otoendoscopic examination, CT scanning of temporal bone after 6 months from the initial surgery, audiological assessment with pure tone 
audiogram after 6 months and a staged second look surgery after 6 months from the initial surgery.

\section{RESULTS}

This study included 20 patients consisted of 14 females $(70 \%)$ and 6 males $(30 \%)$, their ages ranged from 14 to 52 years. The average age at the time of study was $25.9 \pm 9.2$ years. All patients suffered from chronic supportive otitis media with cholesteatoma. Persistent offensive ear discharge and hearing loss (in 9 patient (45\%))were the most common complaints. All patients hadn't undergone previous cholesteatoma surgery. All patients except one were subjected to our surgical technique with the principle of reconstruction of posterior meatal and / or lateral attic walls using the autologous bony

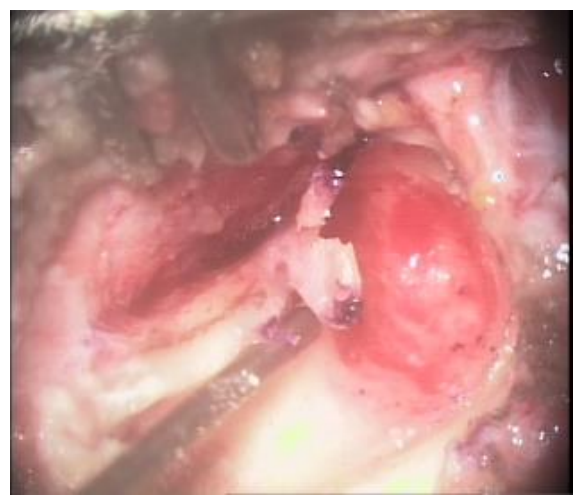

(A) posterosuperior canal wall and bone pâté. The follow - up duration ranged from 7 to 19 months.

Intra operative difficulties: Damage of the bony posterosuperior canal wall during saw cutting occurred in one patient $(5 \%)$, due to excessive thinning of the canal wall during cortical mastoidectomy and the use of straight saw blade applying excess force on the wall, and then CWD procedure was performed. Dural injury during the superior cutting occurred in one patient $(5 \%)$, due to excessive thinning of the dural plate and a relatively large size of the saw blade, and the dural defect was closed with bone wax and gel foam with completion of the surgical procedure. No intraoperative facial nerve injury or sigmoid sinus injury.

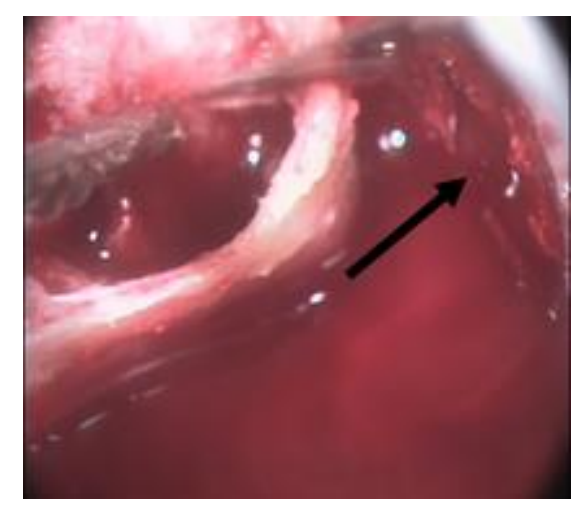

(B)

Figure (5): Canal wall damage during saw cutting(A), dural injury (arrow) during superior cutting(B).

Table (1):Intraoperative difficulties :

\begin{tabular}{lccc}
\hline Intraoperative Difficulty & Total No of cases & $\begin{array}{l}\text { Total No of } \\
\text { complicated cases }\end{array}$ & $\%$ \\
\hline Tympanomeatal flap injury & 20 & 0 & 0 \\
\hline Damage of posterosuperior canal wall & 20 & 1 & $5 \%$ \\
\hline Facial nerve injury & 20 & 0 & 0 \\
\hline Dural injury & 20 & 1 & $5 \%$ \\
\hline Sigmoid sinus injury & 20 & 0 & 0 \\
\hline
\end{tabular}

Through follow up: The duration of follow-up ranged from 7 to 19 months during which: One patient showed infection related signs in the incision site as mild discharge and a transient small skin fistula for about 3 weeks postoperatively, the infection responded to conservative treatment, in the form of secondary sutures, oral antibiotic and topical antibiotic ointment, and then the site of incision remained asymptomatic and dry a long follow up. All patients complained from taste alternation, "metallic taste", due to scarifying of the chorda tympani nerve during surgical procedure, for about 4 to 6 months postoperatively which compensated and disappeared through the period of follow up. Four patients (20\%) complained from mild dizziness for about 2 to 4 months postoperatively which subsided gradually and all of them improved spontaneously without medication. Two patients $(10 \%)$ complained from postoperative tinnitus which persist through the period of follow up and not improved. No facial nerve paralysis was detected in any patient through the period of follow up.

Otoendoscopic follow- up examination revealed that, all but one of the patients exhibited 
a well-healed external ear canal and the healing time was similar to that of CWU surgery. The reconstructed posterior canal wall found to be unimpaired and the new canal appeared near normal in size, shape and contour except in one patient $(5 \%)$, the reconstructed canal wall displaced anteriorly causing mild stenosis of the

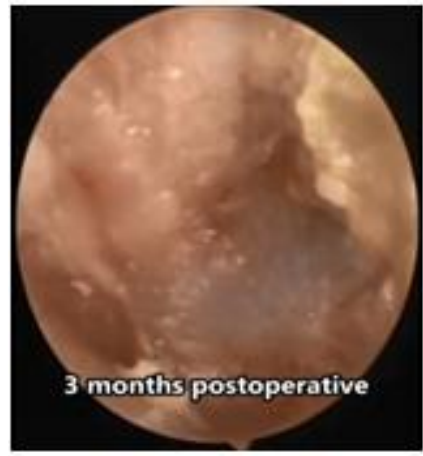

(A) external ear canal. Three patients (15\%) showed the presence of a self-cleaning mesotympanic retraction which remained self-cleaning and stable during follow- up. No postoperative otorrhoea, myringitis, residual perforation in tympanic membrane grafting or extrusion of the bone through the period of follow up.

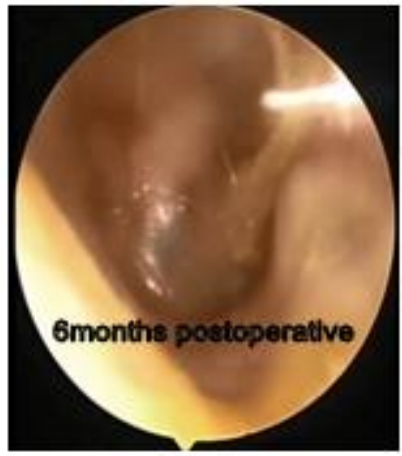

(B)

Figure (6): Postoperative otoendoscopy follow-up (A) after 3 months, (B) after 6 months.

Postoperative follow -up CT scanning was done for all cases after 6 months from the initial surgery and showed that, the posterior canal wall appeared smooth and the newly reconstructed

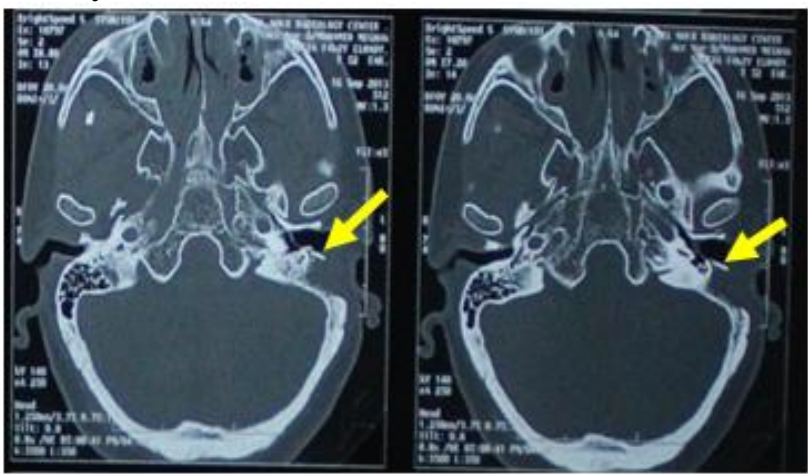

(A)

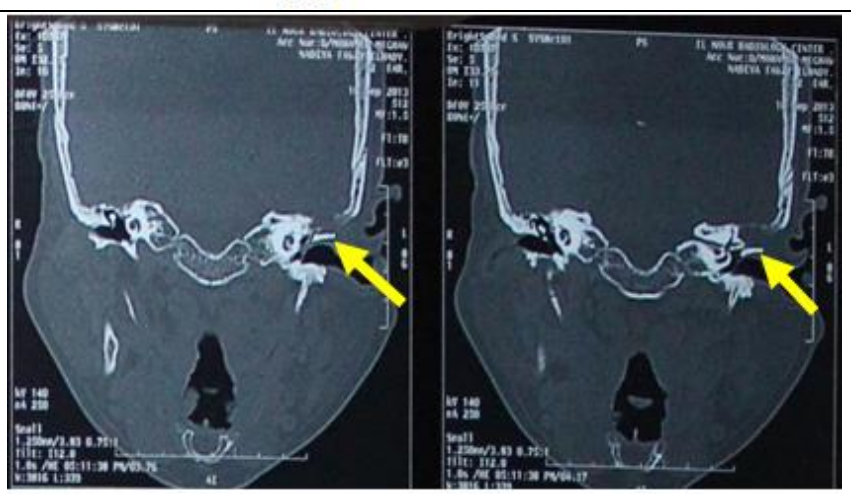

(B)

Figure(7): Postoperative follow-up CT scanning after 6 months showing that the reconstructed wall (arrow) appeared smooth and the new canal appeared near normal in shape and contour, (A) axial plane, (B) coronal plane. canal appeared near normal in shape and contour. No dislocation or necrosis of the reconstructed posterior canal wall had been noted. 
Staged second-look surgery was performed after 6 months from the initial surgery in 15 patients (75\%), 4 patients refused second-look surgery and one patient had CWD procedure during the initial surgery due to damage of the posterosuperior wall during saw cutting. During the staged second-look surgery, the reconstructed posterior canal wall was found to be unimpaired and integrated into the circumjacent bone with near normal contoured ear canal. One patient (5\%) showed recurrent cholesteatoma due to residual in sinus tympani, recurrence was dissected and removed using otoendoscopy. Ossicular reconstructions which had been done in 6 patients (30\%) during the initial surgery were checked and appeared unimpaired.

Table (2): Postoperative complications

\begin{tabular}{lccc}
\hline complication & Total No. of cases & $\begin{array}{c}\text { Total No. of } \\
\text { complicated cases }\end{array}$ & \% \\
\hline Wound infection & 20 & 1 & 5 \\
\hline Taste alternation (metallic taste) & 20 & 20 & 100 \\
\hline Dizziness & 20 & 4 & 20 \\
\hline Tinnitus & 20 & 2 & 10 \\
\hline Mesotympanic retraction & 20 & 3 & 15 \\
\hline Myringitis & 20 & 0 & 0 \\
\hline T.M. perforation & 20 & 0 & 0 \\
\hline Stenosis of EAC & 20 & 1 & 5 \\
\hline Extrusion of bone & 20 & 0 & 0 \\
\hline Recurrent cholesteatoma & 20 & 1 & 5 \\
\hline
\end{tabular}

Audiological findings:

Preoperatively:All patients had preoperative conductive hearing loss except one patient had mixed hearing loss with mild to moderate sensorineural hearing loss (SNHL) 30dBHL. The preoperative $\mathrm{PTA}-\mathrm{ABG}$ ranged from 10 to 45 $\mathrm{dBHL}$ and the average preoperative PTA-ABG was $29.3 \pm 8.5 \mathrm{dBHL}$.

Post-operatively: Pure tone audiogram was done for all cases after 6 months from the initial surgery. Six patients had temporary middle ear effusion which disappeared with medical treatment during follow up.

The patients who had ossicular reconstruction during the initial surgery (6 cases( $30 \%)$ ) , showed improvement of hearing with postoperative PTA-ABG equal or less than 10 $\mathrm{dBHL}$, and the average postoperative PTA-ABG was $8.3 \pm 2.6 \mathrm{dBHL}$.

The patients who did not have ossicular reconstruction during the initial surgery (14 cases $(70 \%)$ ), showed worsening of hearing (more conductive hearing loss), due to ossicular discontinuity, with postoperative PTA-ABG equal or more than $30 \mathrm{dBHL}$ and the average postoperative PTA-ABG was 33.9 $\pm 3.99 \mathrm{dBHL}$.

\section{DISCUSSION}

The main goal of surgery in acquired middle ear cholesteatoma is the complete eradication of disease with an ear free of discharge and inflammation. This can be performed either by open or closed technique (CWD \& CWU mastoidectomy) with their benefits and drawbacks, but there is still an ongoing debate about the best choice (2). Part of the controversy regarding canal wall up versus canal wall down techniques is the potentially higher rate of residual cholesteatoma(up to 60-70\%) with CWU methods - owing to limited exposure of the epi - and retrotympanum -,and the problems of the large mastoid cavity with CWD methods(5).The technique that offers the best of the both worlds (CWD \&CWU procedures) would be one that allows removal of the posterior canal wall to provide adequate exposure for cholesteatoma removal followed by reconstruction of the canal wall for good anatomic and physiologic results(7). The external auditory canal is an important factor in the physiology of the middle ear and sound conductive mechanism. The firm restoration of the tympanic frame and the posterior bony canal wall allows the preservation of the audiological function and resonance of the auditory canal as well as the depth of the middle ear cleft with a deep and (re-) aerated middle ear and a vibrating tympanic membrane. Therefore, the reconstruction of the ear canal must be considered to be an important precondition (8). Various autologous or alloplastic materials have been applied for canal wall reconstruction : Skin of posterior meatal wall(9), Bioactive glass ceramic(ceravital)(10),Hydroxyapatite(11),Titani $\mathrm{m}(\mathbf{1 2})$,Autologous ear Cartilage(13) or Autologous bone grafts from the cortical 
mastoid(2). Our surgical technique - with the principle of cutting the entire posterosuperior bony meatal wall as one piece and its repositioning after complete eradication of cholesteatoma - provides the advantages of CWD and CWU procedures together, as it allows adequate exposure and good visualization for safe removal of cholesteatoma from anterior epitympanum, sinus tympani, on the surface of the facial nerve, the horizontal semicircular canal or around the stapes, avoids the injury of the neighbor structures and has low recurrence rate(5\%), similar to CWD procedure, also, it provides a near normal anatomy of the external ear canal, similar to CWU procedure. In addition, the use of bone pâté in supporting the reconstructed posterior wall and obliteration of the attic and mastoid cavity prevents the postoperative retraction and reduces the mastoid cavity. The described technique is an entirely different one as compared to other techniques of reconstruction, as it offers more advantages over other methods of reconstruction of posterior canal wall. In comparison to the use of prosthetic materials ( Hydroxyapatite, Bioactive glass and Titanium), the use of the autologous bony posterior meatal wall in reconstruction has no danger of immune response and cellular toxicity, no effect on postoperative CT-scanning as well as no extra costs. Our reconstructed bony posterior meatal wall can be evaluated accurately with $\mathrm{CT}-$ scanning which makes it distinct from the autologous ear cartilage which is the most popular material for reconstruction of canal wall (13), because, one of the possible drawbacks after cartilage reconstruction could be the doubtful interpretation of $\mathrm{CT}$ finding which might overestimate pathological finding that was not seen during the consequent revision surgery (14). Asser et al (13), used cartilage and pedicled perichondrial flap for reconstruction of the posterior canal wall, they reported residual cholesteatoma in the tympanic cavity in $12 \%$ of cases; while in this study, only $5 \%$ of cases developed residual cholesteatoma, also, they reported tympanic membrane perforation in $10.3 \%$ of cases; while in this study, no residual tympanic membrane perforation was reported. The bony posterior meatal wall is autologous and orthotopic bone graft, which gives it an advantage over the cortical bone chip used in reconstruction of posterior canal wall (2), which is autologous but non-orthotopic bone graft, and as it was stated that immediate bone healing without interposition of connective tissue is achieved only by immediate firm contact of the bony lid in the surrounding bone if it is in a correct and orthotopic position. Autologous but nonorthotopic bone grafts ought to have deficient contact points in the reconstructed wall, resulting in fibrous and unstable union (15).Gehrking (2), reported that recurrent cholesteatomas were seen in $9.7 \%$ of cases; while in this study, recurrent cholesteatoma was seen in $5 \%$ of cases, also, he observed attic retraction pockets in $6.5 \%$ of cases; while in this study, no attic retraction pockets were observed. However, this described surgical technique has some disadvantages including : relatively longer duration of the procedure, difficulty in cutting the posterosuperior meatal wall by the microsagittal saw and requiring facial recess approach with its risk on the facial nerve, which make this procedure in need for a well skilled and expert surgeon, and as stated by " Sheehy 1988 " (16) ; the key of success in otological surgery is not whose technique one uses, but how well one uses it and one's own ability and judgment.

\section{CONCLUSION}

Reconstruction of posterior meatal and/ or lateral attic walls in cholesteatoma surgery by using the autologous bony posterosuperior meatal wall and bone pâté as described in this study provides adequate intraoperative exposure of all middle ear spaces including anterior epitympanum and sinus tympani, provides a near normal postoperative anatomy of the external ear canal which prevents the postoperative retraction pockets and reduces the mastoid cavity, has a low recurrence rate (5\%) and there are no serious intra- or postoperative complications ,making this surgical procedure an attractive alternative to the standard CWD and CWU procedures.

\section{REFERENCES}

1. Stark T, Gurr A, SudhoffH (2011): Principles of cholesteatoma surgery .HNO 59: 393-399.

2. Gehrking E (2010): Osteoplastic atticoantrotomy with autologous bone chips and a bony attic strut in cholesteatoma surgery. Eur Arch Otorhinolaryngol: 267: 1055-1066.

3. Roland PS, Meyerhoff WL (1999): Opencavity tympanomastoidectomy. Otolaryngol Clinic North Am. 32(2): 525-546.

4. Garham MD, Delap TG, Goldsmith MM (1999): Closed tympanomastoidectomy. Otolaryngol Clinic North Am. 32(3): 547554.

5. Wengen DF, Podvinec M (1998): Fixation of the temporarily removed posterior wall of the auditory canal with plate osteosynthesis: a new technique (in German). Laryngorhinootologie: 77: 70-73. 
6. Tos M (1995): Reconstruction of the posterior ear canal wall. In Tos M (ed) Manual of middle ear surgery. Mastoid surgery and reconstructive procedures, vol 2. Thieme Medical Publishers, Stuttgart: 324-345.

7. Dornhoffer J, Simmons O (2003): Canal wall reconstruction with Mimix hydroxyapatite cement results in an animal model and case study. Laryngoscope:113: 2123-28.

8. Babighian G (2002): Posterior and attic wall osteoplasty: hearing results and recurrence rates in cholesteatoma. OtolNeurotol; 23: 1417.

9. Takahashi H, Hasebe S, Sudo M, et al. (2000): Soft-wall reconstruction for cholesteatoma surgery: Reappraisal. OtolNeurotol; 21 : 2831.

10. Stoor P, Pulkkinen J and Grenman R (2010): Bioactive glass S53P4 in the filling of cavities in the mastoid cell area in Surgery for chronic otitis media. Annals of Otology. Rhinolaryngology; 119(6): 377-382.

11. Estrem SA and HighfillG (1999): Hydroxyapatite canal wall reconstruction mastoid obliteration. Otolaryngol Head Neck Surg; 120: 345-349.

12. Zini C, Quaranta N and Piazza F (2002): Posterior canal wall reconstruction with titanium micro-mesh and bone pâté. Laryngoscope: 112: 753-757.

13. Asser E, Khaled M, Hazem E and Mohsen AR (2010): The use of cartilage and pedicled perichondrial flap for primary reconstruction of the posterior canal wall. Egyptian Journal of Ear,Nose,Throat and Allied Sciences : 11: 75-78.

14. Barbara M (2008): Lateral attic reconstruction technique:preventive surgery for epitympanic retraction pockets. OtolNeurotol; 29 : 522-5.

15. WullsteinHL, WullsteinSR (1990): Principles and definition of tympanoplasty. In: Wullstein HL, Wullstein SR (eds) Tympanoplasty, osteoplastic epitympanotomy. Thieme Medical Publishers, Stuttgart: 32-57.

16. Sheehy J (1988): Cholesteatoma surgery: Canal wall down procedures. Ann OtolRhinolLaryngol;97: 30-35 